\title{
Exterior Electromagnetic Shaping using Wavelet BEM
}

\author{
K. Eppler and H. Harbrecht ${ }^{1}$ \\ 1 TU Chemnitz, Faculty of mathematics
}

Preprint 2003/13

Preprint-Reihe des Instituts für Mathematik

Technische Universität Berlin 
The present paper is concerned with exterior electromagnetic shaping in two dimensions. We model the conductors by regular densities which leads to a finite value for the objective and makes a line-search realizable. In order to compute the pressure on the surface we optimize an Augmented Lagrangian by a Newton method using a second order approach for the Lagrange multiplier. Since the underlying state function satisfies an exterior boundary value problem, we compute its first and second order derivatives by boundary integral equations which are solved numerically by a fast wavelet Galerkin scheme. Numerical results demonstrate that we arrive at a fast and robust algorithm for the solution of the considered class of problems.

AMS subject classification: 49Q10, 65N38 


\title{
EXTERIOR ELECTROMAGNETIC SHAPING USING WAVELET BEM
}

\author{
K. EPPLER AND H. HARBRECHT
}

\begin{abstract}
The present paper is concerned with exterior electromagnetic shaping in two dimensions. We model the conductors by regular densities which leads to a finite value for the objective and makes a line-search realizable. In order to compute the pressure on the surface we optimize an Augmented Lagrangian by a Newton method using a second order approach for the Lagrange multiplier. Since the underlying state function satisfies an exterior boundary value problem, we compute its first and second order derivatives by boundary integral equations which are solved numerically by a fast wavelet Galerkin scheme. Numerical results demonstrate that we arrive at a fast and robust algorithm for the solution of the considered class of problems.
\end{abstract}

\section{INTRODUCTION}

In the present paper we consider a cylindric vertical column of molten liquid metal with planar, simply connected cross section falling down in an electromagnetic field generated by vertical conductors. The frequency of the imposed current is very high such that the magnetic field does not penetrate into the metal and the electromagnetic forces are reduced to the magnetic pressure acting on the interface. In the equilibrium case, a stationary horizontal cross section of fixed volume arises and the three dimensional problem reduces to a simpler two dimensional model.

Seeking the stationary cross section can be reformulated as a shape optimization problem, where the underlying state function is the solution of an exterior boundary value problem for the Laplacian. Introducing a suitable Newton potential, the boundary value problem can be transformed to a boundary integral equation.

Electromagnetic shaping has been addressed in several papers. Novruzi, Pierre, and Roche $[22,23,24]$ considered exterior and interior magnetic shaping by shape optimization via boundary integral equations. Conformal mapping methods have been

Date: June 11, 2003.

Key words and phrases. Exterior electromagnetic shaping, boundary element method, multiscale methods, augmented Lagrangian approach, Newton method, Mårtensson's approach. 
applied by Colaud, Henrot, and Pierre $[2,19]$. Interior shaping without surface tension employing singular integral equations has been treated by Crouzeix, Feat, and Sayas $[3,4]$. The stability of shapes in exterior shaping without surface tension has been investigated in Descloux [10]. To study stablity in a more general context, sufficient optimality conditions for shape problem have been considered in Dambrine and Pierre [8, 9].

In previous papers, the conductors have been modeled by Dirac masses which leads to an infinite objective. We model the conductors via regular densities with radii $\varepsilon>0$. Then, as we will show, the objective is finite, which makes us possible to perform a line-search. Moreover, by the limiting process $\varepsilon \rightarrow 0$, we will provide an explanation for the formal contradiction of an infinte objective and the existence of a regular shape gradient observed in previous papers.

The underlying exterior boundary value problem can be solved efficiently by the boundary element method. We apply a wavelet Galerkin scheme which produces approximate solutions within discretization error accuracy offered by the underlying Galerkin method at a computational expense that stays proportional to the number of unknowns, $[6,15,18,25]$. As it is shown in [13, 14], this results in powerful first and second order shape optimization algorithms.

The Langrange multiplier of the optimization problem corresponds to the pressure on the surface of the liquid metal. Due to its physical meaning we optimize an Augmented Lagrangian by a Newton iteration, which provides a very accurate computation of the Lagrange multiplier. Mårtensson [21] proposed a second order method for the Lagrange multiplier. We provide numerical results which demonstrate that, using using the Mårtensson's approach, the Lagrange multiplier converges faster than in the case of the standard first order method for the multiplier.

The paper is organized as follows. Section 1 is dedicated to the modeling, in particular the formulation of the shape problem and our ansatz for the conductors. In Section 2 we give a brief introduction to the shape calculus. Then, we overview in Section 3 how to derive first and second order derivatives of the state function by boundary integrals equations. Section 4 is concerned with the discretization of the boundary and the wavelet Galerkin scheme. In the last section, that is Section 5, we present our numerical results.

\section{Modeling}

1.1. Model problem. The given configuration satisfies the subsequent physical model. We denote the stationary cross section of the cylindric vertical column of 
liquid metal by $\Omega^{c} \in \mathbb{R}^{2}$ and let $\mathbf{x}=\left[x_{1}, x_{2}\right] \in \mathbb{R}^{2}$. Further, we introduce the following quantities:

- the density current vector $\mathbf{j}_{0}(\mathbf{x})=\left[0,0, j_{0}(\mathbf{x})\right]^{T}$,

- the total magnetic field $\mathbf{B}(\mathbf{x})=\left[B_{1}(\mathbf{x}), B_{2}(\mathbf{x}), 0\right]^{T}$,

- the vacuum permeability $\mu_{0}$,

- the surface tension $\sigma$,

- the outer unit normal vector $\mathbf{n}=\left[n_{1}, n_{2}\right]^{T}$ of $\Omega^{c}$,

- the curvature $\kappa$ of $\Gamma=\partial \Omega^{c}$, and

- the volume $V\left(\Omega^{c}\right)=\left|\Omega^{c}\right|$ of the liquid metal.

Then, in case of the quilibrium, the cross section of the liquid metal and the related magnetic field satisfy the system of equations

$$
\begin{aligned}
\nabla \times \mathbf{B} & =\mu_{0} \mathbf{j}_{0} & & \text { in } \Omega:=\mathbb{R}^{2} \backslash \Omega^{c}, \\
\nabla \cdot \mathbf{B} & =0 & & \text { in } \Omega, \\
\mathbf{B} \cdot\left[\begin{array}{c}
\mathbf{n} \\
0
\end{array}\right] & =0 & & \text { on } \Gamma, \\
\frac{\|\mathbf{B}\|^{2}}{2 \mu_{0}}+\sigma \kappa & =p & & \text { constant on } \Gamma, \\
\mathbf{B} & =\mathcal{O}\left(\|\mathbf{x}\|^{-2}\right) & & \text { as }\|\mathbf{x}\| \rightarrow \infty,
\end{aligned}
$$

provided that $\Omega^{c}$ fulfils $V\left(\Omega^{c}\right)=V_{0}$. The unknowns of the problem are the boundary $\Gamma=\partial \Omega$ and the pressure constant $p$ in the equilibrium condition (1.4).

1.2. Reformulation as a shape optimization problem. Introducing a scalar potential $U: \Omega \mapsto \mathbb{R}$, condition (1.2) implies the ansatz

$$
\mathbf{B}(\mathbf{x})=\nabla \times\left[\begin{array}{c}
0 \\
0 \\
u(\mathbf{x})
\end{array}\right]=\left[\begin{array}{c}
\frac{\partial U}{\partial x_{2}}(\mathbf{x}) \\
-\frac{\partial U}{\partial x_{1}}(\mathbf{x}) \\
0
\end{array}\right] .
$$

From the boundary condition (1.3) we deduce $\partial U / \partial \mathbf{t} \equiv 0$, where $\mathbf{t}=\mathbf{n}^{\perp}=$ $\left[n_{2},-n_{1}\right]^{T}$ denotes the tangent vector. This yields $U \equiv$ const. on $\Gamma$. For sake of convenience we choose const. $=0$. Hence, in view of (1.1) and (1.5), the scalar potential $U$ has to satisfy

$$
\begin{aligned}
-\Delta U & =\mu_{0} j_{0} & & \text { in } \Omega, \\
U & =0 & & \text { on } \Gamma, \\
U & =\mathcal{O}(1) & & \text { as }\|\mathbf{x}\| \rightarrow \infty, \\
\nabla U & =\mathcal{O}\left(\|\mathbf{x}\|^{-2}\right) & & \text { as }\|\mathbf{x}\| \rightarrow \infty .
\end{aligned}
$$

Let us define the total energy of the system by

$$
\widehat{E}(\Omega):=\widehat{J}(\Omega)+\sigma P(\Omega),
$$


where

$$
\widehat{J}(\Omega):=-\frac{1}{2 \mu_{0}} \int_{\Omega}\|\nabla U\|^{2} d \mathbf{x}, \quad P(\Omega)=\int_{\Gamma} 1 d \sigma_{\mathbf{x}} .
$$

Note that $P(\Omega)$ is the perimeter of the domain $\Omega^{c}$. Then, the equilibrium domain $\Omega^{\star}$ can be viewed as a stationary point of the shape functional (1.6) under the constraint that $V\left(\Omega^{c}\right)=V_{0}$ be imposed. Being more precise, for sufficiently regular domains and data, it holds the following necessary condition.

Theorem 1.1 (Necessary condition). If $\Omega^{\star}$ is optimal, then there exists $a \lambda^{\star} \neq 0$ such that

$$
\left.\left\{\frac{\|\nabla U\|^{2}}{2 \mu_{0}}+\sigma \kappa-\lambda^{\star}\right\}\right|_{\Gamma^{\star}} \equiv 0 \quad \text { and } \quad\left|\Omega^{\star}\right|=V\left(\Omega^{\star}\right)=V_{0}
$$

Proof. First, the Lagrangian is introduced as usual

$$
L(\Omega, \lambda):=E(\Omega)-\lambda\left(V(\Omega)-V_{0}\right)
$$

Applying, for example, a shape calculus by a smooth nontangential boundary perturbation field $\mathbf{d} \in\left(C^{2}(\Gamma)\right)^{2}$, i.e., $\Gamma_{t}=\Gamma+t \mathbf{d}$, the solution $U_{t}$ and $E\left(\Omega_{t}\right)$ are well defined if $t$ is sufficiently small. Using standard results (cf. [28]), we arrive at the following expression for the directional derivative

$$
d L(\Omega, \lambda)[\mathbf{d}]=\lim _{t \rightarrow 0} \frac{L\left(\Omega_{t}, \lambda\right)-L(\Omega, \lambda)}{t}=\int_{\Gamma}\langle\mathbf{d}, \mathbf{n}\rangle\left\{\frac{\|\nabla U\|^{2}}{2 \mu_{0}}+\sigma \kappa-\lambda\right\} d \sigma_{\mathbf{x}} .
$$

The claim $d L(\Omega, \lambda)[\mathbf{d}]=0$ for all nontrivial $\mathbf{d}$ yields the desired result.

Remark 1.2. The necessary condition is just the equilibrium condition (1.4). Consequently, the Lagrange multiplier $\lambda^{\star}$ corresponds to the unknown pressure constant p. Moreover, a stable equilibrium satisfies the sufficient optimality condition.

We assume that the magnetic field is generated by vertical live wires at the position $\mathbf{x}_{i}$ and of the amperage $\alpha_{i} I$ each, $i=1, \ldots, M$. We investigate two concepts of modeling such wires, namely, either Dirac masses $\alpha_{i} I \delta\left(\mathbf{x}_{i}\right)$ or as regular density, namely $\alpha_{i} I /\left(\pi \varepsilon^{2}\right) \chi_{B_{\varepsilon}\left(\mathbf{x}_{i}\right)}$. Herein, $I$ denotes the unity of amperage and

$$
B_{\varepsilon}\left(\mathbf{x}_{i}\right):=\left\{\mathbf{x} \in \mathbb{R}^{2}:\left\|\mathbf{x}-\mathbf{x}_{i}\right\| \leq \varepsilon\right\}
$$

Of course, any piecewise constant and compactly supported density $j_{0}$ provides a finite energy and sufficient regularity for the existence of a shape gradient. Since the modeling by Dirac masses leads to an infinite energy, we proceed the present paper with a regular density and will consider Dirac masses as the limit $\varepsilon \rightarrow 0$ as far as possible. 
We simplify the formulation of the quantities as follows. We set $A:=2 \sigma /\left(\mu_{0} I^{2}\right)$, $j=j_{0} / I$, and $u:=U /\left(\mu_{0} I\right)$. Hence, the scaled total energy is given by

$$
E(\Omega)=J(\Omega)+A P(\Omega)
$$

with

$$
J(\Omega)=-\int_{\Omega}\|\nabla u\|^{2} d \mathbf{x}
$$

Consequently, determining the free surface is equivalent to the following shape optimization problem

$$
E(\Omega) \rightarrow \min _{\Omega \subseteq \mathbb{R}^{2}} \quad \text { subject to } \quad V\left(\Omega^{c}\right)=V_{0}
$$

with

$$
V\left(\Omega^{c}\right)=\int_{\Omega^{c}} 1 d \mathbf{x}
$$

1.3. Newton potential for circular conductors. The exterior boundary value problem with respect to $u$ can be reformulated as a boundary integral equation if we find a suitable Newton potential $N_{j} \in C^{1,1}(\mathbb{R})$ satisfying the equation

$$
\begin{aligned}
-\Delta N_{j} & =j & & \text { in } \mathbb{R}^{2} \\
N_{j} & =\mathcal{O}(1) & & \text { as }\|\mathbf{x}\| \rightarrow \infty \\
\nabla N_{j} & =\mathcal{O}\left(\|\mathbf{x}\|^{-2}\right) & & \text { as }\|\mathbf{x}\| \rightarrow \infty
\end{aligned}
$$

Then, the ansatz

$$
u=N_{j}+v
$$

yields the problem of seeking a harmonic function $v$ satisfying

$$
\begin{aligned}
-\Delta v & =0 & & \text { in } \Omega, \\
v & =-N_{j} & & \text { on } \Gamma, \\
v & =\mathcal{O}(1) & & \text { as }\|\mathbf{x}\| \rightarrow \infty, \\
\nabla v & =\mathcal{O}\left(\|\mathbf{x}\|^{-2}\right) & & \text { as }\|\mathbf{x}\| \rightarrow \infty .
\end{aligned}
$$

Lemma 1.3. Assume $\sum_{i=1}^{M} \alpha_{i}=0$ and consider $M$ circular conductors with finite diameter $\varepsilon$, that is

$$
j(\mathbf{x})=\sum_{i=1}^{M} \frac{\alpha_{i}}{\pi \varepsilon^{2}} \chi_{B_{\varepsilon}\left(\mathbf{x}_{i}\right)}, \quad \mathbf{x} \in \mathbb{R}^{2} .
$$

Then, a Newton potential $N_{j} \in C^{1,1}(\mathbb{R})$ satisfying (1.10) is given by

$$
N_{j}=\sum_{i=1}^{M} N_{i j}, \text { where } N_{i j}(\mathbf{x})= \begin{cases}\frac{\alpha_{i}}{4 \pi}\left[1-\frac{\left\|\mathbf{x}-\mathbf{x}_{i}\right\|^{2}}{\varepsilon^{2}}-2 \log \varepsilon\right], & \mathbf{x} \in B_{\varepsilon}\left(\mathbf{x}_{i}\right), \\ -\frac{\alpha_{i}}{2 \pi} \log \left\|\mathbf{x}-\mathbf{x}_{i}\right\|, & \mathbf{x} \notin B_{\varepsilon}\left(\mathbf{x}_{i}\right),\end{cases}
$$


Proof. For the $i$-th conductor we make the ansatz

$$
N_{i j}(\mathbf{x})= \begin{cases}c_{i}-\frac{\alpha_{i}\left\|\mathbf{x}-\mathbf{x}_{i}\right\|^{2}}{4 \pi \varepsilon^{2}}, & \text { if } \mathbf{x} \in B_{\varepsilon}\left(\mathbf{x}_{i}\right) \\ d_{i} \log \left\|\mathbf{x}-\mathbf{x}_{i}\right\|, & \text { if } \mathbf{x} \notin B_{\varepsilon}\left(\mathbf{x}_{i}\right)\end{cases}
$$

with unknown constants $c_{i}, d_{i} \in \mathbb{R}$. The claim $N_{j}=\sum_{i=1}^{M} N_{i j} \in C^{1,1}(\mathbb{R})$ yields the equations

$$
c_{i}-\frac{\alpha_{i}}{4 \pi}=d_{i} \log \varepsilon, \quad-\frac{\alpha_{i}}{2 \pi \varepsilon}=\frac{d_{i}}{\varepsilon}, \quad i=1, \ldots, M
$$

that is

$$
c_{i}=\frac{\alpha_{i}}{2 \pi}\left[\frac{1}{2}-\log \varepsilon\right], \quad d_{i}=-\frac{\alpha_{i}}{2 \pi}, \quad i=1, \ldots, M .
$$

If and only if $\sum_{i=1}^{M} \alpha_{i}=0$ there holds

$$
\lim _{\|\mathbf{x}\| \rightarrow \infty} N_{j}(\mathbf{x})=\lim _{\|\mathbf{x}\| \rightarrow \infty} \sum_{i=1}^{M} \alpha_{i} \log \left\|\mathbf{x}-\mathbf{x}_{i}\right\|=\lim _{\|\mathbf{x}\| \rightarrow \infty} \sum_{i=1}^{M} \alpha_{i} \log \frac{\left\|\mathbf{x}-\mathbf{x}_{i}\right\|}{\|\mathbf{x}\|}
$$

and, hence, $N_{j}=\mathcal{O}\left(\|\mathbf{x}\|^{-1}\right)$ and $\left\|\nabla N_{j}\right\|=\mathcal{O}\left(\|\mathbf{x}\|^{-2}\right)$ as $\|\mathbf{x}\| \rightarrow \infty$.

Remark 1.4. If $\sum_{i=1}^{M} \alpha_{i} \neq 0$ we conclude $N_{j}=\mathcal{O}(\log \|\mathbf{x}\|)$ and $\left\|\nabla N_{j}\right\|=\mathcal{O}\left(\|\mathbf{x}\|^{-1}\right)$ as $\|\mathbf{x}\| \rightarrow \infty$, i.e., the Newton potential decays not sufficiently fast. This is obvious from the physical point of view, since the over-all amperage has to be zero.

Note that both, the behaviour at infinity as well as $N_{j}$ in a neighbourhood of $\Gamma$ is independent of $\varepsilon$ provided that $\min _{i=1}^{M} \operatorname{dist}\left(\Omega^{c}, \mathbf{x}_{i}\right)>\varepsilon$.

1.4. Computation of the objective. First, we prove the following lemma which is helpful if we consider circluar conductors with diameter $\varepsilon$ tending to 0 .

Lemma 1.5. Given a regular density $j$ according to (1.13), then there holds

$$
J(\Omega)=\int_{\Omega}-j(\mathbf{x}) u(\mathbf{x}) d \mathbf{x}=\int_{\Omega}-j(\mathbf{x}) N_{j}(\mathbf{x}) d \mathbf{x}+\int_{\Gamma} N_{j}(\mathbf{x}) \frac{\partial u}{\partial \mathbf{n}}(\mathbf{x}) d \sigma,
$$

where $\mathbf{n}$ is the inner normal with respect to the exterior domain $\Omega$.

Proof. We define $\Omega_{R}=\Omega \cap B_{R}(\mathbf{0})$ with a sufficiently large $R>0$. Using $u=v+N_{j}$ and applying Green's formula repeatedly leads to

$$
\begin{aligned}
J(\Omega) & =\lim _{R \rightarrow \infty} \int_{\Omega_{R}}-\|\nabla u(\mathbf{x})\|^{2} d \mathbf{x} \\
& =\lim _{R \rightarrow \infty}\left\{\int_{\Omega_{R}}-j(\mathbf{x}) u(\mathbf{x}) d \mathbf{x}-\int_{\partial \Omega_{R}} u(\mathbf{x}) \frac{\partial u}{\partial \mathbf{n}}(\mathbf{x}) d \sigma\right\} \\
& =\lim _{R \rightarrow \infty}\left\{\int_{\Omega_{R}}-j(\mathbf{x}) N_{j}(\mathbf{x}) d \mathbf{x}-\int_{\partial \Omega_{R}} N_{j}(\mathbf{x}) \frac{\partial u}{\partial \mathbf{n}}(\mathbf{x})+u(\mathbf{x}) \frac{\partial v}{\partial \mathbf{n}}(\mathbf{x}) d \sigma\right\}
\end{aligned}
$$


that is the assertion since $j$ is compactly supported and (1.10), (1.12) imply

$$
u(\mathbf{x})=\mathcal{O}(1), \quad \frac{\partial u}{\partial \mathbf{n}}=\mathcal{O}\left(\|\mathbf{x}\|^{-2}\right), \quad \text { as }\|\mathbf{x}\| \rightarrow \infty
$$

Next, we compute the first part in more detail and consider the conductors tending to Dirac masses, that is $\varepsilon \rightarrow 0$. Inserting (1.13) and (1.14), we get for the domain integral on the right hand side of (1.15)

$$
\begin{aligned}
\int_{\operatorname{supp} j} N_{j}(\mathbf{x}) j(\mathbf{x}) d \mathbf{x} & =\sum_{i=1}^{M} \int_{B_{\varepsilon}\left(\mathbf{x}_{i}\right)} \sum_{k=1}^{M} N_{i j}(\mathbf{x}) \cdot \frac{\alpha_{i}}{\pi \varepsilon^{2}} d \mathbf{x} \\
& =\sum_{i=1}^{M} \int_{B_{\varepsilon}\left(\mathbf{x}_{i}\right)} \sum_{k=1}^{M}\left[N_{i j}(\mathbf{x})-\sum_{\substack{k=1 \\
k \neq i}}^{M} \frac{\alpha_{k}}{2 \pi} \log \left\|\mathbf{x}-\mathbf{x}_{k}\right\|\right] \frac{\alpha_{i}}{\pi \varepsilon^{2}} d \mathbf{x} \\
& =T_{1}+T_{2}+T_{3} .
\end{aligned}
$$

Herein, the first term $T_{1}$ represents the infinite part of the energy

$$
T_{1}=-\sum_{i=1}^{M} \int_{B_{\varepsilon}\left(\mathbf{x}_{i}\right)} \frac{\alpha_{i}^{2}}{2 \pi^{2} \varepsilon^{2}} \log \varepsilon d \mathbf{x}=-\log \varepsilon \sum_{i=1}^{M} \frac{\alpha_{i}^{2}}{2 \pi} \stackrel{\varepsilon \rightarrow 0}{\longrightarrow} \infty,
$$

while the remaining terms $T_{2}$ and $T_{3}$ represent the finite parts

$$
\begin{gathered}
T_{2}=\sum_{i=1}^{M} \frac{\alpha_{i}^{2}}{4 \pi} \int_{B_{\varepsilon}\left(\mathbf{x}_{i}\right)} \frac{\alpha_{i}^{2}}{4 \pi^{2} \varepsilon^{2}}\left[1-\frac{\left\|\mathbf{x}-\mathbf{x}_{i}\right\|^{2}}{\varepsilon^{2}}\right] d \mathbf{x}=\frac{1}{8 \pi} \sum_{i=1}^{M} \alpha_{i}^{2}, \\
T_{3}=-\sum_{i=1}^{M} \sum_{\substack{k=1 \\
k \neq i}}^{M} \frac{\alpha_{i} \alpha_{k}}{2 \pi^{2} \varepsilon^{2}} \int_{B_{\varepsilon}\left(\mathbf{x}_{i}\right)} \log \left\|\mathbf{x}-\mathbf{x}_{k}\right\| d \mathbf{x} \stackrel{\varepsilon \rightarrow 0}{\longrightarrow}-\sum_{i=1}^{M} \sum_{\substack{k=1 \\
k \neq i}}^{M} \frac{\alpha_{i} \alpha_{k}}{2 \pi^{2}} \log \left\|\mathbf{x}_{i}-\mathbf{x}_{k}\right\| .
\end{gathered}
$$

By neglecting $T_{1}$ there remains a finite energy for $\varepsilon=0$ (Dirac masses)

$$
J(\Omega)=\frac{1}{8 \pi} \sum_{i=1}^{M} \alpha_{i}^{2}-\sum_{i=1}^{M} \sum_{\substack{k=1 \\ k \neq i}}^{M} \frac{\alpha_{i} \alpha_{k}}{2 \pi} \log \left\|\mathbf{x}_{i}-\mathbf{x}_{k}\right\|-\int_{\Gamma} N_{j}(\mathbf{x}) \frac{\partial u}{\partial \mathbf{n}}(\mathbf{x}) d \sigma .
$$

Nevertheless, a more promising consequence is presented in the next lemma.

Lemma 1.6. The shape gradient at an arbitrary admissible domain is independent of $\varepsilon$ provided that $\min _{i=1}^{M} \operatorname{dist}\left(\Omega^{c}, \mathbf{x}_{i}\right)>\varepsilon$. In particular, the shape gradient exists in a limiting sense even in the case of Dirac masses, i.e., for $\varepsilon=0$, despite the fact of an infinite energy.

Proof. Consider the conductors in the positions $x_{i}$ with amperage $\alpha_{i}$ and the domain $\Omega$ fixed. By taking into account (1.14), (1.15), Remark 1.4 and the above considerations, one confirms that $\varepsilon$ occurs only in expressions which do not depend on 
the actual shape. Hence, there is no influence in the difference $E\left(\Omega_{t}\right)-E(\Omega)$ for arbitrary regular perturbed domains $\Omega_{t}\left(|t| \leq t_{0}\right)$ and, consequently, no influence in the shape gradient and higher order shape derivatives. For $\varepsilon=0$, the limiting shape gradient might be viewed as the shape gradient of the finite part of the energy.

Remark 1.7. The formal contradiction between the existence of a regular shape gradient and an infinite energy in the case of Dirac masses was already observed and discussed in [24]. Nevertheless, the above Lemma provides a more complete explanation for that interesting fact. Moreover, based on the above results, a linesearch is realizable in the optimization algorithms.

\section{Shape calculus}

2.1. Boundary variation. The outer security set of the shape problem (1.8) is given as $D=\mathbb{R}^{2}$ (the hold all set). We consider as admissible domains all domains $\Omega \subseteq D \subseteq \mathbb{R}^{2}$ such that $\Omega^{c}$ is a bounded domain of smoothness $C^{2, \alpha}, \alpha>0$, and star-shaped with respect to $B_{\delta}(\mathbf{0})$.

It is rather convenient to identify the domain $\Omega \in C^{2, \alpha}$ with a parametric representation of its boundary $\Gamma=\partial \Omega$. Due to the star-shapedness this parametric representation can be chosen in polar coordinates

$$
\Gamma:=\left\{\gamma(\phi)=r(\phi)\left[\begin{array}{c}
\cos \phi \\
\sin \phi
\end{array}\right]: \phi \in[0,2 \pi]\right\},
$$

where $r \in C_{\text {per }}^{2, \alpha}[0,2 \pi]$ is a positive function with $r>\delta$ and

$$
C_{\text {per }}^{2, \alpha}[0,2 \pi]=\left\{r \in C^{2, \alpha}[0,2 \pi]: r^{(i)}(0)=r^{(i)}(2 \pi), i=0,1,2\right\} .
$$

As standard variation for perturbed domains $\Omega_{\varepsilon}$ and boundaries $\Gamma_{\varepsilon}$, respectively, we introduce a function $d r \in C_{\text {per }}^{2, \alpha}[0,2 \pi]$

$$
r_{\varepsilon}(\phi)=r(\phi)+\varepsilon d r(\phi)
$$

where $\gamma_{\varepsilon}(\phi)=r_{\varepsilon}(\phi) \mathbf{e}_{r}(\phi)$ is always a Jordan curve. Herein, $\mathbf{e}_{r}(\phi)=[\cos \phi, \sin \phi]^{T}$ denotes the unit vector in the inner radial direction of $\Omega$.

The main advantage of this simple approach is a complete embedding of the shape problem into a Banach space setting. That is, both the shapes and its increments, can be viewed as elements of $C_{\text {per }}^{2, \alpha}[0,2 \pi]$.

2.2. Shape derivatives. Next, we adopt the shape calculus developed in $[11,12]$ to our shape optimization problem. Based on this shape calculus, the assertion of Theorem 1.1 can be derived as well. 
Remark 2.1. In [14] we considered the minimization of functionals of the form

$$
J(\Omega)=\int_{\Omega} h(\mathbf{x}) u(\mathbf{x}) d \mathbf{x} \rightarrow \min
$$

with a sufficiently smooth function $h$ and the state function $u$ satisfying the Poisson equation with some Dirichlet data. Due to (1.15), the abstract framework of [14] can be applied if we consider the case of finite diameter of the conductors $(\varepsilon>0)$, since a piecewise constant and compactly supported $j$ is regular enough for performing the shape calculus in an exterior domain. Moreover, the treatment of the perimeter in the energy functional (1.6) is straightforward.

According to (1.14) and (1.15) the objective is computed via

$$
E(\Omega)=\int_{\Gamma}\left[\frac{\partial u}{\partial \mathbf{n}} N_{j}+A\right] d \sigma+C
$$

where $C=C\left(\varepsilon, \alpha_{1}, \ldots, \alpha_{M}, \mathbf{x}_{1}, \ldots, \mathbf{x}_{M}\right)$. Consequently, the shape gradient of this functional reads

$$
\nabla E(\Omega)[d r]=\int_{0}^{2 \pi} d r r\left\{\left[\frac{\partial u}{\partial \mathbf{n}}\right]^{2}-A \kappa(\phi)\right\} d \phi
$$

The boundary integral representation of the shape Hessian is given by

$$
\begin{aligned}
\nabla^{2} E(\Omega)\left[d r_{1}, d r_{2}\right] & =\int_{0}^{2 \pi} d r_{1} d r_{2} \frac{\partial u}{\partial \mathbf{n}}\left\{\frac{\partial u}{\partial \mathbf{n}}+\frac{r^{2}}{\sqrt{r^{2}+r^{\prime 2}}} \frac{\partial^{2} u}{\partial \mathbf{n}^{2}}+\frac{r r^{\prime}}{\sqrt{r^{2}+r^{\prime 2}}} \frac{\partial^{2} u}{\partial \mathbf{n} \partial \mathbf{t}}\right\} \\
& -\frac{2 r}{\sqrt{r^{2}+r^{\prime 2}}} d r_{1} \frac{\partial u}{\partial \mathbf{n}} \cdot \frac{\partial d u\left[d r_{2}\right]}{\partial \mathbf{n}} \\
& +\frac{A}{\sqrt{r^{2}+r^{\prime 2}}}\left\{r^{2} d r_{1}^{\prime} d r_{2}^{\prime}+d r_{1} d r_{2} \frac{\left(2{r^{\prime}}^{2}-r^{2}\right)\left(r^{\prime 2}-r r^{\prime \prime}\right)}{r^{2}+r^{\prime 2}}\right\} d \phi .
\end{aligned}
$$

Herein, $d u\left[d r_{2}\right]$ denotes the local shape derivative of the state function which solves

$$
\begin{aligned}
\Delta d u & =0 & & \text { in } \Omega, \\
d u & =d r_{2}\left\langle\mathbf{e}_{r}, \mathbf{n}\right\rangle \frac{\partial u}{\partial \mathbf{n}} & & \text { on } \Gamma .
\end{aligned}
$$

Note that the last term on the right hand side of (2.19) implies implies the $H^{1}$ coercivity of the hessian.

With respect to the volume one finds the derivates

$$
\nabla V\left(\Omega^{c}\right)[d r]=\int_{0}^{2 \pi} d r r d \phi, \quad \nabla^{2} V\left(\Omega^{c}\right)\left[d r_{1}, d r_{2}\right]=\int_{0}^{2 \pi} d r_{1} d r_{2} d \phi .
$$




\section{BOUNDARY INTEGRAL FORMULATION}

In order to compute the shape functional and its gradient we require only the normal derivative $\partial u / \partial \mathbf{n}$. Hence, its knowledge is sufficient to perform a first order optimization method. But the computation of the Hessian requires also second order derivatives, namely $\partial^{2} u / \partial \mathbf{n}^{2}$ and $\partial^{2} u /(\partial \mathbf{n} \partial \mathbf{t})$.

The ansatz (1.11) leads to the normal derivative $\partial u / \partial \mathbf{n}$ according to

$$
\frac{\partial u}{\partial \mathbf{n}}=\frac{\partial v}{\partial \mathbf{n}}+\frac{\partial N_{j}}{\partial \mathbf{n}}
$$

with the Newton potential $N_{j}$ defined in (1.14) and $v$ satisfying the boundary value problem (1.12). We introduce the modified single layer operator $\mathcal{V}$ and the double layer operator $\mathcal{K}$ defined by

$$
\begin{aligned}
& (\mathcal{V} u)(\mathbf{x}):=-\frac{1}{2 \pi} \int_{\Gamma} \log \|\mathbf{x}-\mathbf{y}\|\left\{u(\mathbf{y})-\int_{\Gamma} \frac{u(\mathbf{z})}{|\Gamma|} \sigma_{\mathbf{z}}\right\} d \sigma_{\mathbf{y}}+\int_{\Gamma} \frac{u(\mathbf{z})}{|\Gamma|} \sigma_{\mathbf{z}}, \\
& (\mathcal{K} u)(\mathbf{x}):=\frac{1}{2 \pi} \int_{\Gamma} \frac{\left\langle\mathbf{n}_{\mathbf{y}}, \mathbf{x}-\mathbf{y}\right\rangle}{\|\mathbf{x}-\mathbf{y}\|^{2}} u(\mathbf{y}) d \sigma_{\mathbf{y}} .
\end{aligned}
$$

Then, the normal derivative of $v$ is given by the Dirichlet-to-Neumann map

$$
\mathcal{V} \frac{\partial v}{\partial \mathbf{n}}=-\left(\frac{1}{2}-\mathcal{K}\right) N_{j}
$$

Remark 3.1. The modification of the single layer operator yields a solution $\partial v / \partial \mathbf{n}$ satisfying $\int_{\Gamma} \partial v / \partial \mathbf{n} d \sigma=0, c f .[20]$. This ensures $v(\mathbf{x}) \rightarrow 0$ and $\|\nabla v(\mathbf{x})\|=\mathcal{O}\left(\|\mathbf{x}\|^{-2}\right)$ as $\|\mathbf{x}\| \rightarrow \infty$.

If we denote the function space of all squared integrable functions on $\Gamma$ with respect to the canonical inner product by $L^{2}(\Gamma)$ and the associated Sobolev spaces by $H^{s}(\Gamma)$, $s \in \mathbb{R}$, then, in this context, $\mathcal{V}: H^{-1 / 2}(\Gamma) \rightarrow H^{1 / 2}(\Gamma)$ defines an operator of the order -1 while $\frac{1}{2}-\mathcal{K}: H^{1 / 2}(\Gamma) \rightarrow H^{1 / 2}(\Gamma)$ defines an operator of the order 0 .

Next, according to [14], due to zero Dirichlet boundary conditions, we find the equation

$$
\frac{\partial^{2} u}{\partial \mathbf{n}^{2}}=\kappa\left(\frac{\partial N_{j}}{\partial \mathbf{n}}-\frac{\partial v}{\partial \mathbf{n}}\right)-j,
$$

that is $\frac{\partial^{2} u}{\partial \mathbf{n}^{2}}$ can be derived directly from the first order derivative $\partial v / \partial \mathbf{n}$. Finally, from (1.11) we deduce

$$
\frac{\partial^{2} u}{\partial \mathbf{n} \partial \mathbf{t}}=\frac{\partial^{2} v}{\partial \mathbf{n} \partial \mathbf{t}}+\frac{\partial^{2} N_{j}}{\partial \mathbf{n} \partial \mathbf{t}}
$$

with the unknown function $\partial^{2} v /(\partial \mathbf{n} \partial \mathbf{t})$. It is advantageous to choose again a boundary integral formulation since we do not loose the regularity of $\partial v / \partial \mathbf{n}$. In accordance 
with $[14,26,27]$, let the operators

$$
\begin{aligned}
& {\left[\mathcal{V}, \frac{\partial}{\partial \mathbf{t}}\right]: H^{-1 / 2}(\Gamma) } \rightarrow H^{1 / 2}(\Gamma), \\
& {\left[\frac{1}{2}-\mathcal{K}, \frac{\partial}{\partial \mathbf{t}}\right]: H^{1 / 2}(\Gamma) \rightarrow H^{1 / 2}(\Gamma) . }
\end{aligned}
$$

denote the commutators of $\mathcal{V}$ and $\frac{1}{2}-\mathcal{K}$. Then, differentiation of (3.21) with respect to the tangent vector yields the boundary integral equation

$$
\mathcal{V} \frac{\partial^{2} v}{\partial \mathbf{n} \partial \mathbf{t}}=\left(\frac{1}{2}-\mathcal{K}\right) \frac{\partial N_{j}}{\partial \mathbf{t}}+\left[\frac{1}{2}-\mathcal{K}, \frac{\partial}{\partial \mathbf{t}}\right] N_{j}-\left[\mathcal{V}, \frac{\partial}{\partial \mathbf{t}}\right] \frac{\partial v}{\partial \mathbf{n}}
$$

\section{Discretization}

4.1. Finite dimensional representation of boundaries. Since the infinite dimensional optimization problem cannot be solved directly, we replace it by an finite dimensional problem. Based on the polar coordinate approach, we can express the smooth function $r \in C_{\text {per }}^{2, \alpha}([0,2 \pi])$ by the Fourier series

$$
r(\phi)=a_{0}+\sum_{n=1}^{\infty} a_{n} \cos n \phi+a_{-n} \sin n \phi .
$$

Hence, it is reasonable to take the truncated Fourier series

$$
r_{N}(\phi)=a_{0}+\sum_{n=1}^{N} a_{n} \cos n \phi+a_{-n} \sin n \phi .
$$

as approximation of $r$. We mention that also other boundary representations like Bsplines can be considered as well. The advantages of our approach is an exponential convergence $r_{N} \rightarrow r$ if the shape is analytical.

Since $r_{N}$ has the $2 N+1$ degrees of freedom $a_{-N}, a_{1-N}, \ldots, a_{N}$, we arrive at a finite dimensional optimization problem in the open set

$$
A_{N}:=\left\{a_{-N}, a_{1-N}, \ldots, a_{N} \in \mathbb{R}: r_{N}(\phi)>0, \phi \in[0,2 \pi]\right\} \subset \mathbb{R}^{2 N+1} .
$$

Then, via the identification $r_{N} \Leftrightarrow \Omega_{N}$, the finite dimensional approximation of problem (1.8) reads as

$$
E\left(\Omega_{N}\right) \rightarrow \min \quad \text { subject to } \quad V\left(\Omega_{N}^{c}\right)=V_{0}
$$

The associated gradients and hessians have to be computed with respect to all directions $d r, d r_{1}, d r_{2}=\cos N \phi, \cos (N-1) \phi, \ldots, \sin (N-1) \phi, \sin N \phi$. 
4.2. The wavelet Galerkin scheme. Boundary element methods provide a common tool for the solution of boundary integral equations. In general, cardinal Bsplines are used as ansatz functions in the Galerkin formulation. But discretizing the boundary integral equations (3.21) and (3.22) with respect to such single-scale bases yields densely populated system matrices. In combination with the ill-posedness of the single layer operator and its commutator, the solution is of at least quadratic complexity.

The crucial idea of the wavelet Galerkin scheme is a change of bases, i.e., applying appropriate wavelet bases instead of the traditional single-scale bases. On the one hand, based on the well known norm equivalences of wavelet bases, the diagonals of the system matrices define optimal preconditioners, cf. [5, 7, 25]. On the other hand, the resulting quasi-sparse system matrices can be compressed without loss of accuracy such that the complexity for the solution of the boundary integral equations becomes linear, cf. [6, 16, 17, 18].

For a fixed domain, we have to solve the boundary integral equations (3.21) and (3.22) several times, namely, the Dirichlet-to-Neumann map (3.21) applies to the state function and the associated local shape derivatives, while (3.22) has to be evaluated for the state function. Hence, an efficient realization discretizes both, the boundary integral operators on the left and on the right hand side of the given boundary integral equations. This requires a mixed formulation in order to achieve the optimal order of convergence.

For sake of convenience, we choose the parametrical representation of the boundary in accordance with

$$
\gamma:[0,1] \rightarrow \Gamma, \quad s \mapsto \gamma(s):=r(2 \pi s)\left[\begin{array}{l}
\cos (2 \pi s) \\
\sin (2 \pi s)
\end{array}\right]
$$

On the level $l$, we subdivide the interval $[0,1]$ equidistantly into $2^{l}$ intervals. On this partition, we consider 1-periodic piecewise constant $(d=1)$ and piecewise linear $(d=2)$ spline wavelets as constructed in [1] which we denote by $\bar{\Psi}_{L}^{(d)}:=\left\{\bar{\psi}_{l, k}^{(d)}\right\}$. Such wavelets provide vanishing moments of order $\widetilde{d}$, that is

$$
\int_{\mathbb{R}} \bar{\psi}_{l, k}^{(d)}(s) s^{\alpha} d s=0, \quad 0 \leq \alpha<\widetilde{d} .
$$

According to $[16,17]$, it suffices to consider piecewise constant wavelets with $\widetilde{d}=3$ vanishing moments and piecewise linear wavelets with $\widetilde{d}=2$ vanishing moments. Setting

$$
\Psi_{L}^{(d)}:=\left\{\bar{\psi}_{l, k}^{(d)} \circ \gamma^{-1}\right\}
$$


we obtain (curved) piecewise constant and linear wavelet bases on the boundary $\Gamma$. It is well known that the bases $\Psi_{L}^{(d)}$ are uniformly stable in $H^{-1 / 2}(\Gamma)$ and $H^{1 / 2}(\Gamma)$, respectively, cf. [1, 29]. We refer the reader to [1, 18, 25] for further details.

We make the ansatz $\partial v / \partial \mathbf{n}=\Psi_{L}^{(1)} \mathbf{v}_{L}$ and $\partial^{2} v /(\partial \mathbf{n} \partial \mathbf{t})=\Psi_{L}^{(1)} \widetilde{\mathbf{v}}_{L}$. Then, introducing the system matrices

$$
\begin{array}{ll}
\mathbf{V}_{L}:=\left(\mathcal{V} \Psi_{L}^{(1)}, \Psi_{L}^{(1)}\right)_{L^{2}(\Gamma)}, & \widetilde{\mathbf{V}}_{L}:=\left(\left[\mathcal{V}, \frac{\partial}{\partial \mathbf{t}}\right] \Psi_{L}^{(1)}, \Psi_{L}^{(1)}\right)_{L^{2}(\Gamma)}, \\
\mathbf{K}_{L}:=\left(\left(\frac{1}{2}-\mathcal{K}\right) \Psi_{L}^{(2)}, \Psi_{L}^{(1)}\right)_{L^{2}(\Gamma)}, & \widetilde{\mathbf{K}}_{L}:=\left(\left[\frac{1}{2}-\mathcal{K}, \frac{\partial}{\partial \mathbf{t}}\right] \Psi_{L}^{(2)}, \Psi_{L}^{(1)}\right)_{L^{2}(\Gamma)},
\end{array}
$$

the data vectors

$$
\mathbf{u}_{L}:=\left(N_{j}, \Psi_{L}^{(2)}\right)_{L^{2}(\Gamma)}, \quad \widetilde{\mathbf{u}}_{L}:=\left(\frac{\partial N_{j}}{\partial \mathbf{t}}, \Psi_{L}^{(2)}\right)_{L^{2}(\Gamma)}
$$

and the mass matrix

$$
\mathbf{G}_{L}:=\left(\Psi_{L}^{(2)}, \Psi_{L}^{(2)}\right)_{L^{2}(\Gamma)},
$$

the boundary integral equation (3.21) corresponds to

$$
\mathbf{V}_{L} \mathbf{v}_{L}=-\mathbf{K}_{L}\left(\mathbf{G}_{L}\right)^{-1} \mathbf{u}_{L}
$$

while (3.22) corresponds to

$$
\mathbf{V}_{L} \widetilde{\mathbf{v}}_{L}=\mathbf{K}_{L}\left(\mathbf{G}_{L}\right)^{-1} \widetilde{\mathbf{u}}_{L}+\widetilde{\mathbf{K}}_{L}\left(\mathbf{G}_{L}\right)^{-1} \mathbf{u}_{L}+\widetilde{\mathbf{V}}_{L} \mathbf{v}_{L}
$$

Consequently, we have to compute only four system matrices for each new domain, but solving the discrete systems several times with different data vectors (see subsection 4.1). We mention that $\left(\mathbf{G}_{L}\right)^{-1} \mathbf{u}_{L} \Psi_{L}^{(d)}$ and $\left(\mathbf{G}_{L}\right)^{-1} \widetilde{\mathbf{u}}_{L} \Psi_{L}^{(d)}$ denote the $L^{2}$ othogonal projections of the given Dirichlet data $N_{j}$ and $\partial N_{j} / \partial \mathbf{t}$ onto the space of the piecewise linears.

Theorem 4.1. Let the domain $\Omega$ be fixed and sufficiently smooth. Then, the computational expense for solving (3.21) and (3.22) by the wavelet Galerkin scheme stays proportional to the number of unknowns.

Assuming further the directions $d r, d r_{1}, d r_{2}$ sufficiently smooth and fixed, the discretization errors are given by

$$
\begin{gathered}
|(E-\widehat{E})(\Omega)|=\mathcal{O}\left(h_{L}^{3}\right), \\
|(\nabla E-\widehat{\nabla E})(\Omega)[d r]|=\mathcal{O}\left(h_{L}^{2}\right), \\
\left|(\Delta E-\widehat{\Delta E})(\Omega)\left[d r_{1}, d r_{2}\right]\right|=\mathcal{O}\left(h_{L}^{2}\right),
\end{gathered}
$$

where $h_{L}=2^{-L}$ denotes the step width on the level $L$ and $\widehat{E}, \widehat{\nabla E}, \widehat{\Delta E}$ indicate the approximate solutions.

Proof. The first statement is a consequence of $[6,16,17,18]$. The second statement is proven in [14]. 


\section{Algorithms And nUmerical Realization}

5.1. The wavelet BEM solver. In this subsection we like to demonstrate the power of our BEM solver for the computation of the shape functional $E(\Omega)$, its gradient $\nabla E(\Omega)$ and its hessian $\nabla^{2} E(\Omega)$.

We choose the domain $\Omega$ as an ellipse with semiaxies $1 / 2$ and 1 which we approximate via 33 Fourier coefficients $(N=16)$. On the level $L$, we compute the solutions $E_{L}(\Omega), \nabla E_{L}(\Omega) \in \mathbb{R}^{33}$ and $\nabla E_{L}(\Omega) \in \mathbb{R}^{33 \times 33}$ with respect to all Fourier coefficients in accordance with Subsection 4.1. In Table 5.1 we tabulate their relative errors measured with respect to the associated Euclidean norms. As reference solution we use the solutions on the level $L=14$, that is $2^{L}=16384$ piecewise constant and linear boundary elements each. The bracketed values indicate the ratio of the previous error and the present error. A cubic order of convergence implies a ratio of 8 while for a quadratic order the ratio should be close to four. One figures out of Table 5.1 rates of convergence as predicted in Theorem 4.1, i.e., a cubic order with respect to the shape functional and quadratic orders with respect to its gradient and Hessian.

\begin{tabular}{|c|c|c|c|c|c|}
\hline$L$ & $2^{L}$ & $\frac{\left|\left(E_{14}-E_{L}\right)(\Omega)\right|}{E_{14}(\Omega) \mid}$ & $\frac{\left\|\left(\nabla E 14-\nabla E_{L}\right)(\Omega)\right\|}{\left\|\nabla E_{14}(\Omega)\right\|}$ & $\frac{\left\|\left(\nabla^{2} E_{14}-\nabla^{2} E_{L}\right)(\Omega)\right\|}{\left\|\nabla^{2} E_{14}(\Omega)\right\|}$ & cpu-time \\
\hline 5 & 32 & $1.9 \mathrm{e}-03$ & $5.1 \mathrm{e}-02$ & $8.1 \mathrm{e}-01$ & $0.5 \mathrm{sec}$. \\
6 & 64 & $2.8 \mathrm{e}-04(6.7)$ & $5.5 \mathrm{e}-03(9.3)$ & $1.0 \mathrm{e}-01(8.1)$ & 0.9 sec. \\
7 & 128 & $2.0 \mathrm{e}-05(14)$ & $7.1 \mathrm{e}-04(7.7)$ & $5.9 \mathrm{e}-03(17)$ & $1.8 \mathrm{sec}$. \\
8 & 256 & $1.3 \mathrm{e}-06(16)$ & $1.7 \mathrm{e}-04(4.3)$ & $9.3 \mathrm{e}-04(6.3)$ & 4.1 sec. \\
9 & 512 & $2.2 \mathrm{e}-07(5.7)$ & $4.2 \mathrm{e}-05(4.0)$ & $2.7 \mathrm{e}-04(3.4)$ & 9.5 sec. \\
10 & 1024 & $8.0 \mathrm{e}-09(28)$ & $1.0 \mathrm{e}-05(4.0)$ & $7.5 \mathrm{e}-05(3.6)$ & 21 sec. \\
11 & 2048 & $2.5 \mathrm{e}-09(3.2)$ & $2.6 \mathrm{e}-06(4.1)$ & $1.9 \mathrm{e}-05(3.9)$ & 50 sec. \\
12 & 4096 & $1.3 \mathrm{e}-09(2.0)$ & $6.2 \mathrm{e}-07(4.2)$ & $4.7 \mathrm{e}-06(4.1)$ & 113 sec. \\
13 & 8192 & $1.1 \mathrm{e}-11(110)$ & $1.2 \mathrm{e}-07(5.0)$ & $9.6 \mathrm{e}-07(4.9)$ & 288 sec. \\
\hline
\end{tabular}

TABLE 5.1. Relative errors of approximation and over-all computing times.

The last column of Table 5.1 contains the over-all computing times to derive the measured quantities. Unfortunately, the ascent of the computing times is only nearly linear. We suppose that this results from cache-based effects of the computer. But let us remark that, on the level $L=13$, we compute four system matrices and solve two linear systems (with a lot of different right hand sides) in less than five minutes.

5.2. Augmented Lagrangian Approach. On the one hand, it is well known that the pure Lagrangian functional is not useful, in particular if the optimal Lagrange multiplier is not known in advance. On the other hand, a simple penalty method is 
neither very accurate nor does it compute the Lagrange multiplier which corresponds to the pressure. Hence, we consider an Augmented Lagrangian method for equality constraints

$$
L_{c}(\Omega, \lambda)=E(\Omega)-\lambda\left(V\left(\Omega^{c}\right)-V_{0}\right)+\frac{c}{2}\left(V\left(\Omega^{c}\right)-V_{0}\right)^{2} .
$$

Of course, a stationary domain of $L_{c}\left(\Omega, \lambda^{\star}\right)$ satisfies the necessary condition of Theorem 1.1 derived from the pure Lagrangian functional (1.7), and vice versa.

The optimization algorithm for the Augmented Lagrangian method reads as follows:

- initialization: choose initial guesses $\lambda^{(0)}$ and $\Omega^{(0)}$ for $\lambda^{\star}$ and $\Omega^{\star}$, respectively, - inner iteration: solve $L_{c}\left(\Omega, \lambda^{(n)}\right) \rightarrow \min$ with initial guess $\Omega^{(n)}$,

- outer iteration: update $\lambda^{(n+1)}=\lambda^{(n)}-c\left(V\left(\Omega^{c}\right)-V_{0}\right)$ and choose $\Omega^{(n+1)}$ as the solution from the inner iteration.

In the inner iteration, we employ a Newton scheme as described in the previous sections combined with a quadratic line-search. In order to ensure a reliably value for the Lagrange multiplier, ten outer iterations are performed. Note that if the Euclidean norm of the gradient of (5.26) is larger than 5, we perform only quasi Newton steps. We compute six different examples as specified in Figure 5.1. Herein, the positions and the amperages of the conductors can be found. The parameters $V_{0}$ and $A$ are chosen according to Table 5.2.

The numerical setting is as follows. We approximate the boundary via 65 Fourier coefficients, that is the choice $N=32$ according to (4.23). Therefore, the approximate gradient is a vector $\in \mathbb{R}^{65}$ and the Hessian a matrix $\in \mathbb{R}^{65 \times 65}$. The boundary integral equations (3.21) and (3.22) are discretized using $512(L=9)$ piecewise constant and linear boundary elements each. Consequently, we pay tribute to the more complicated domains and double the number of Fourier coefficients as well as BEM unknowns in comparison to $[13,14]$.

We start the optimization process always with the circle of volume $V_{0}$ centered in $\mathbf{0}$ and $\lambda^{(0)}=0$. Note that the choice $c=50$ is required to achieve convergence of the Newton method. Setting $c$ substantially smaller than 50 yields divergence of the second order optimization algorithm. In the last three columns of Table 5.2, we list the over-all number of Newton iterations, the final Euclidean norm of the gradient of (5.26) and the computed Lagrange multiplier.

5.3. Mårtensson's approach. As we have shown in [14], the Newton scheme makes a line-search nearly obsolete in comparison to first order methods. As a consequence, the bottleneck during the iteration process is now the slow (first order) approach of the Lagrange multiplier. 

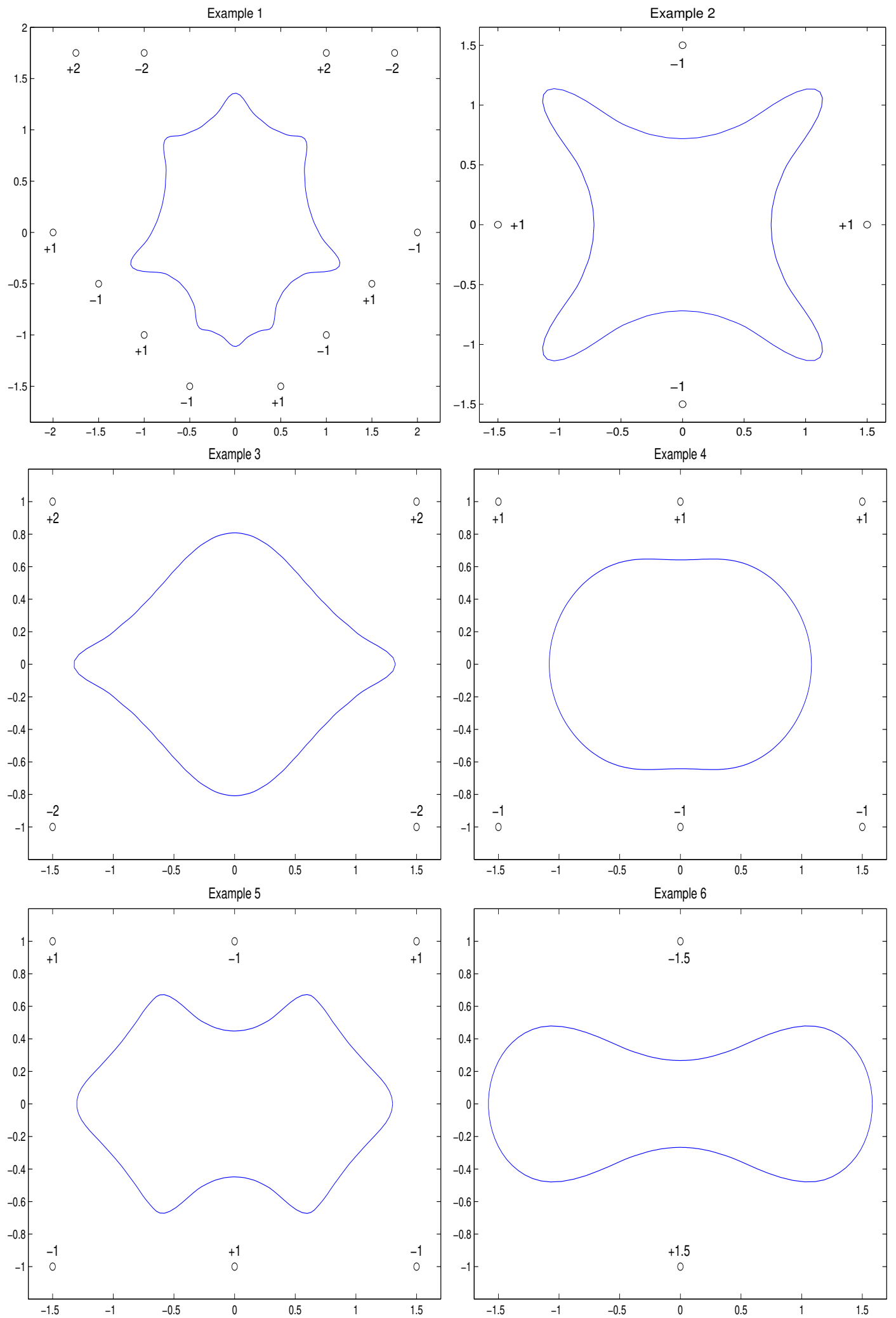

Figure 5.1. Examples 1-6 (from left to right and top to bottom). 


\begin{tabular}{|c|c|c|c|c|c|c|}
\hline Example & $V_{0}$ & $A$ & iterations & $\left\|\nabla L_{c}(\Omega, \lambda)\right\|$ & $\lambda$ & cpu-time \\
\hline 1 & $\pi$ & 0.001 & 27 & $2.171 \mathrm{e}-13$ & $3.010 \mathrm{e}-02$ & 163 sec. \\
2 & $\pi$ & 0.007 & 36 & $3.471 \mathrm{e}-12$ & $7.573 \mathrm{e}-02$ & 215 sec. \\
3 & $3 \pi / 4$ & 0.010 & 29 & $2.007 \mathrm{e}-12$ & $3.541 \mathrm{e}-01$ & 141 sec. \\
4 & $3 \pi / 4$ & 0.500 & 39 & $2.843 \mathrm{e}-09$ & $9.738 \mathrm{e}-01$ & 138 sec. \\
5 & $3 \pi / 4$ & 0.010 & 40 & $3.129 \mathrm{e}-13$ & $8.856 \mathrm{e}-02$ & 262 sec. \\
6 & $3 \pi / 4$ & 0.100 & 41 & $5.071 \mathrm{e}-07$ & $2.191 \mathrm{e}-01$ & 202 sec. \\
\hline
\end{tabular}

TABLE 5.2. Setting and results of Examples 1-6 using the Augmented Lagrangian functional.

Mårtensson [21] proposed a second order method for the Lagrange multiplier. More precisely, the multiplier is seen as a function depending on the primal variable, i.e., the actual domain $\Omega$, namely

$$
\lambda=\lambda(\Omega)=\frac{\left\langle\nabla V\left(\Omega^{c}\right), \nabla E(\Omega)\right\rangle}{\left\langle\nabla V\left(\Omega^{c}\right), \nabla V\left(\Omega^{c}\right)\right\rangle} .
$$

Instead of the constant multiplier in the shape functional (5.26), a first order Taylor expansion of the above multiplier function, developed at the given initial guess $\Omega^{(n)}$, is inserted into the Augmented Lagrangian functional, that is

$$
\begin{aligned}
L_{c}^{(n)}(\Omega) & =E(\Omega)-\left[\lambda\left(\Omega^{(n)}\right)+\nabla \lambda\left(\Omega^{(n)}\right) \cdot\left(\Omega-\Omega^{(n)}\right)\right]\left(V\left(\Omega^{c}\right)-V_{0}\right) \\
& +\frac{c}{2}\left(V\left(\Omega^{c}\right)-V_{0}\right)^{2} .
\end{aligned}
$$

Herein, the difference $\Omega-\Omega^{(n)}$ has to be understood as the difference of the associated Fourier coefficients. Of course, the gradient and the hessian are slightly different compared to those of (5.26), but the computation is straightforward and all ingredients appear also in the first and second order derivatives of (5.26).

Using Mårtensson's approach, the optimization algorithm reads as follows:

- initialization: choose an initial guess $\Omega^{(0)}$ for $\Omega^{\star}$

- inner iteration: solve $L_{c}^{(n)}(\Omega) \rightarrow$ min with initial guess $\Omega^{(n)}$,

- outer iteration: choose $\Omega^{(n+1)}$ as the solution from the inner iteration.

Likewise as in the previous subsection, we employ a Newton scheme combined with a quadratic line-search in the inner iteration. But now we stop the outer iteration if the norm of the gradient of the functional (5.28) is less than 1e-6.

We underly the set-up of the optimization algorithm identically to that in the previous subsection, except the penalty parameter is chosen considerably smaller since $c=50$ yields divergence. It turns out that $c=5$ is a good choice. We remark that 


\begin{tabular}{|c|c|c|c|c|}
\hline Example & iterations & $\left\|\nabla L_{c}^{(n)}\left(\Omega, \Omega^{(n)}\right)\right\|$ & $\lambda$ & cpu-time \\
\hline 1 & 13 & $1.593 \mathrm{e}-08$ & $3.010 \mathrm{e}-02$ & 89 sec. \\
2 & 14 & $1.483 \mathrm{e}-07$ & $7.573 \mathrm{e}-02$ & 89 sec. \\
3 & 15 & $4.544 \mathrm{e}-08$ & $3.541 \mathrm{e}-01$ & 102 sec. \\
4 & 15 & $2.117 \mathrm{e}-07$ & $9.738 \mathrm{e}-01$ & 101 sec. \\
5 & 21 & $4.092 \mathrm{e}-07$ & $8.856 \mathrm{e}-02$ & 162 sec. \\
6 & 24 & $3.257 \mathrm{e}-07$ & $2.191 \mathrm{e}-01$ & 199 sec. \\
\hline
\end{tabular}

TABLE 5.3. Results of Examples 1-6 using Mårtensson's approach.

we do not have any reasonable explanation why $c$ depends such strong on the chosen approach for the Lagrange multiplier. As the results in Table 5.3 confirm, the convergence of the optimization algorithm is accelerated considerably without compromising the accuracy. The number of iterations is about $50 \%$ less in comparison with the first order approach. The costs for Mårtensson's approach are negligible compared to the saved computing time resulting from the reduced number of iterations.

\section{REFERENCES}

[1] A. Cohen, I. Daubechies, and J.-C. Feauveau. Biorthogonal bases of compactly supported wavelets. Pure Appl. Math., 45:485-560, 1992.

[2] O. Colaud and A. Henrot. Numerical approximation of a free boundary problem arising in electromagnetic shaping. SIAM J. Numer. Anal.. 31: 1109-1127, 1994.

[3] M. Crouzeix. Variational approach of magnetic shaping problem. Eur. J. Mech, B/Fluids, 10: 527-536, 1991.

[4] M. Crouzeix, Ph. Feat and F.-J. Sayas. Theoretical and numerical study of a free boundary problem by boundary integral methods M2AN, vol. 35, no.6, p. 1137-1158, 2001.

[5] W. Dahmen. Wavelet and multiscale methods for operator equations. Acta Numerica, 6:55-228, 1997.

[6] W. Dahmen, H. Harbrecht and R. Schneider. Compression techniques for boundary integral equations - optimal complexity estimates. Preprint SFB 393/02-06, TU Chemnitz, 2002. submitted to SIAM J. Numer. Anal.

[7] W. Dahmen and A. Kunoth. Multilevel preconditioning. Numer. Math., 63:315-344, 1992.

[8] M. Dambrine and M. Pierre. About stability of equilibrium shapes. M2AN 34, No.4, 811-834 (2000).

[9] M. Dambrine. On variations of the shape Hessian and sufficient conditions for the stability of critical shapes. RACSAM, Rev. R. Acad. Cienc. Exactas Fs. Nat., Ser. A Mat. 96, No.1, 95-121 (2002) 
[10] J. Descloux. Stability of solutions of the bidimensional magnetic shaping problem in absense of surface tension. Eur. J. Mech, B/Fluids, 10: 513-526, 1991.

[11] K. Eppler. Boundary integral representations of second derivatives in shape optimization. Discussiones Mathematicae (Differential Inclusion Control and Optimization), 20:63-78, 2000.

[12] K. Eppler. Optimal shape design for elliptic equations via BIE-methods. J. of Applied Mathematics and Computer Science, 10:487-516, 2000.

[13] K. Eppler and H. Harbrecht. Numerical solution of elliptic shape optimization problems using wavelet-based BEM. Optim. Methods Softw., 18:105-123, 2003.

[14] K. Eppler and H. Harbrecht. 2nd Order Shape Optimization using Wavelet BEM. Preprint 06-2003, TU Berlin, 2003. submitted to Optim. Methods Softw.

[15] H. Harbrecht. Wavelet Galerkin schemes for the boundary element method in three dimensions. PHD Thesis, Technische Universität Chemnitz, Germany, 2001.

[16] H. Harbrecht, F. Paiva, C. Pérez, and R. Schneider. Biorthogonal wavelet approximation for the coupling of FEM-BEM. Numer. Math., 92:325-356, 2002.

[17] H. Harbrecht, F. Paiva, C. Pérez, and R. Schneider. Wavelet preconditioning for the coupling of FEM-BEM. Num. Lin. Alg. Appl., 10:197-222, 2003.

[18] H. Harbrecht and R. Schneider. Wavelet Galerkin Schemes for 2D-BEM. In Operator Theory: Advances and Applications, volume 121. Birkhäuser, (2001).

[19] A. Henrot and M. Pierre About existence of equilibria in electromagnetic casting. $Q$. Appl. Math. 49, No.3, 563-575 (1991).

[20] R. Kress. Linear Integral Equations. Springer-Verlag, Berlin-Heidelberg, 1989.

[21] K. Mårtensson (1973) A new approach to constrained function optimization. J. of Optimization Theory and Application. 12: 531-554.

[22] A. Novruzi Contribution en Optimisation des Formes et Applications. PHD Thesis, Nancy, 1997.

[23] A. Novruzi and J.-R. Roche. Second derivatives, Newton method, application to shape optimization. INRIA-report No. 2555 (1995)

[24] M. Pierre and J.-R. Roche Computation of free surfaces in the electromagnetic shaping of liquid metals by optimization algorithms. Eur. J. Mech, B/Fluids, 10: 489-500, 1991.

[25] R. Schneider. Multiskalen- und Wavelet-Matrixkompression: Analysisbasierte Methoden zur Lösung großer vollbesetzter Gleichungssyteme. B.G. Teubner, Stuttgart, 1998.

[26] H. Schulz, C. Schwab and W.L. Wendland. An extraction technique for boundary element methods. In W. Hackbusch and G. Wittum, editors, Boundary Elements: Implementation and Analysis of Advanced Algorithms, pages 219-231, 1996.

[27] C. Schwab and W.L. Wendland. On the extraction technique in boundary integral equations. Math. Comp., 68:91-122, 1999.

[28] J. Sokolowski and J.-P. Zolesio. Introduction to Shape Optimization. Springer, Berlin, 1992. 
[29] L. Villemoes. Wavelet analysis of refinement equations. SIAM J. Math. Anal., 25:1433-1460, 1994.

Institut für Mathematik, Technische Universität Berlin, 10623 Berlin, GerMANY.

E-mail address: eppler@math.tu-berlin.de

Fakultät für Mathematik, Technische Universität Chemnitz, 09107 ChemNitz, Germany.

E-mail address: helmut.harbrecht@mathematik.tu-chemnitz.de 